\title{
Examples of the application of light-tissue interaction to biomedical engineering
}

\author{
A. Cysewska-Sobusiak, A. Hulewicz, Z. Krawiecki \\ \& G. Wiczynski \\ Poznan University of Technology, \\ Institute of Electrical Engineering and Electronics, Poland
}

\begin{abstract}
The subject of this paper relates to the measurable effects of light behavior in biological materials. The problems considered include some current topics concerning the achievements and still ongoing development in the selected fields of light-tissue interaction to be used in biomedical measurements. Measurable effects of interaction between light and tissues may be utilized in biomedicine with emission, reflection or transmission modes, respectively. The latter is especially considered in this paper: transillumination as the method of examination by the passage of light through tissues or a body cavity is a diagnostic technique in the course of intensive development at the moment. Red light and near-infrared radiation emitted by high-efficient LEDs can be noninvasively transmitted through the blood-supplied tissues. Some representative examples of the medical applications of non-invasive effects of light-tissue interaction are discussed. Under the transillumination and illumination from underneath, it is possible to diagnose and monitor the parameters of tissues and organs examined. This paper includes discussion of selected issues related to the biophysical and optical phenomena used and examples of practical applications of tissue transillumination techniques. The authors briefly report the current state-in-the-art and also present their own results. Among other things, the presented examples include: optoelectronic techniques used in monitoring of living tissue vitality, and promising results obtained during preliminary experiments with transillumination scanning applied to fingers of the human hand.
\end{abstract}

Keywords: biomedical optics, light-tissue interaction, optoelectronic sensors, noninvasive biomeasurements, medical imaging. 


\section{Introduction}

The different parts of the electromagnetic spectrum have very different effects upon interaction with biological objects. Radiation energy may be transmitted, reflected, absorbed and scattered by living tissues but light is first of all absorbed strongly. The optical part of the whole spectrum offers a wide range of measurement possibilities. The role of optical techniques in current medical measurements and imaging is especially significant [1-5]. Using optoelectronic sensing allows us to convert sophisticated effects of light-biological object interaction to electrical signals, which may be convenient to process and measure. The-state-of-the-art, taken here into account as the background, refers to the reported optical parameters of tissues when exposed to light of wavelengths included in the optical window in tissue spectrum $[1,6]$.

When a biological object is exposed to illumination, we can receive the selective optical response to particular wavelengths. Information about optical parameters of the medium is included in this response, however, other physical parameters of a given object also influence its output signal. The surface conditions, internal structure and size in the direction of light action are of great importance.

In the paper, discussion is focused on the selected methods which apply the effects of light-tissue interaction to be used in noninvasive biomedical engineering. The examples presented by the authors include: optoelectronic techniques used in monitoring of the living tissues vitality, and promising results obtained during preliminary experiments with transillumination scanning applied to human hand fingers. Previous own authors' experience in one-dimensional modeling for transmission pulse oximetry $[7,8]$ has been adapted.

\section{Interdisciplinary aspects of biomedical engineering}

The discipline of biomedical engineering is a diverse field that includes such areas as e.g. bioinstrumentation, biomaterials, medical imaging, biosensors, biotechnology, and tissue engineering [1]. This interdisciplinary field based in both engineering and the life sciences relates to physiological as well as electrical, optical, mechanical, chemical, and other principles to be applied in diagnostics and therapy. Over the last years, the development of new and different medical equipment is impressive.

Advances in medical and biological technology are due to the increasing interaction and collaboration between medial and engineering scientists. There is also necessity to work well in a well-integrated interdisciplinary team. Efficient and safe application of light-tissue interactions to biomedical engineering must meet the various human and technical needs such as: medical practice, ethics, clinical care of patients, instrumentation reliability, material biocompatibility. 


\section{Specificity of interaction between light and living tissues}

Among the applied methods of tissue parameters measurement, a tendency to develop methods based on detection and analysis of natural and forced biooptical phenomena is significant. Bio-signals can be acquired in a variety of ways. Bio-optical signals are generated by the optical attributes of biological systems. There is a specific window extending from $600 \mathrm{~nm}$ to $1200 \mathrm{~nm}$ in which optical radiation can penetrate into human tissue set and propagate through it. However, due to strong scattering of the light, the practical implementation of optical transillumination for medical imaging is a difficult task. Measurements are very rich in artefacts, noises and disturbances which affect processing and can cause the decrease in useful output signals.

Tissues are optically turbid media that are highly scattering. A typical scattering coefficient for visible light in human tissues is $100 \mathrm{~cm}^{-1}$ in comparison with $0.2 \mathrm{~cm}^{-1}$ for $\mathrm{X}$ rays used in medical diagnostics.

The interactions occurring between the light and the tissues result in scattering, absorption and fluorescence, providing information on the structure, physiology, biochemistry and molecular functions. Optical imaging is used for description of surface and volume structures.

Reports in the literature often give the particular information about parameters, e.g., percentage transmission or reflection of light measured at the particular wavelength penetrating a particular human or animal organ $[1,2,6]$. Many reports refer to data collected in vitro or in vivo, but always invasively. Inhomogeneities in the living complex structures must lead to a spread in measured values when compared to those obtained for the selected homogeneous samples. A particular tissue composition very depends on blood and water contents what results in differences between values of optical parameters if to determine them at several compositions of various kinds and size. There are several sources of optical inhomogeneity of a biological object, which can be described mainly as structural imperfections and optical anisotropy. Depending on the locality and diameter of the light beam passing through the object the effects of light-tissue interaction can differ very much. The specific human blood feature is that hemoglobin which is carrying oxygen (oxyhemoglobin $\mathrm{HbO}_{2}$ ) absorbs light in the IR region of the spectrum; hemoglobin that isn't carrying oxygen (reduced hemoglobin $\mathrm{Hb}$ ) absorbs visible red light [2].

\subsection{Optoelectronic sensors}

By definition, an optoelectronic sensor is a device that produces an electrical signal proportional to the amount of light incident on its active area [3, 4]. These sensors still become more efficient, portable and smaller.

Measurable effects of interaction between light and tissues may be used in biomedical engineering with emission, reflection or transmission mode, respectively. The methods used in positioning objects in relation to the sources and optical radiation sources are presented in fig. 1. 
a)

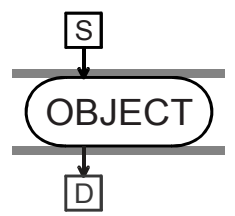

b)

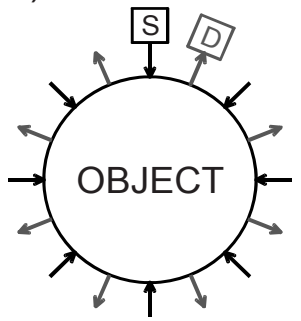

c)

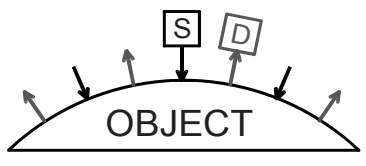

Figure 1: Examples of the variants of the investigated object position in relation to optoelectronic sensors containing the photoemitters (light sources S) and photodetectors D [9].

Optoelectronic sensors usually combine a set of LEDs emitting the incident light $(600 \mathrm{~nm}$ to $1000 \mathrm{~nm})$ as the photoemitter with a broadband photodiodes $(350 \mathrm{~nm}$ to $1100 \mathrm{~nm})$ as the photodetector of transmitted or reflected light. Modern high-efficient LEDs are very useful as moderately narrowband photoemitters with an approximately Gaussian spectral shape. The silicon PIN photodiodes may be used in practice as photodetectors. These small and lightweight elements are very sensitive, have low noise levels, and convert the light into electronic signal linearly, in the whole measuring window and over a large dynamic range.

A current driving LEDs intensity makes the sensor to be 'smart' by means to control levels of the transmitted light and provide incident light which might be transmitted through various real objects, as being more or less thick and pigmented. Evidently, a way the photoemitter is driving by current impulses as well as the selective photocurrent is converted, amplified and conditioned, are also critical $[4,5]$.

The sensor types include: light-to-voltage converters, light-to-frequency converters, ambient light sensors, linear sensor arrays, colour sensors, reflective light sensors [3]. The transmission variants of light-tissue interaction (fig. 1a) that is accomplished with the object transillumination can be often more convenient and sensitive than the reflection variants.

\subsection{Advantages of transillumination}

Transillumination is understood as the phenomenon of transmitting optical radiation with defined parameters by a given object, which becomes the carrier of information about the characteristics of this object.

Continuous monitoring of light transmitted in the penetrated region could offer a control about the changes inside the objects, and become important in predicting the light action outcome. Representative examples of transillumination may include:

- detection of body fluids components (spectrophotometry, oximetry in vivo),

- localization of veins, cysts, and neoplasms from underneath, 
- transillumination with white light (instead of X-rays),

- imaging and monitoring of pulse wave (photoplethysmography),

- transmission variant of pulse oximetry,

- optical tomography.

Optical properties of transilluminated or illuminated tissues or organs depends on strong light absorption and scattering - numerous issues related to the result interpretation still remain unsolved. A given set of living tissues consists of many components, which create the determined spatial configuration. Light transmitted through tissues is classified into three categories: ballistic light, quasi-ballistic light, and diffuse light. Depending on the locality and diameter of the light beam passing through the object the effects of light-tissue interaction can differ very much. Optical radiation that is to play the role of an effective information carrier should be sufficiently coherent and, due to the high optical density of the object, should also have possibly high intensity. However, for higher power density quantities, some destructive photothermal effects can occur. Wavelength and power of radiation selected improperly may not only act ineffectively, but may cause damage or destruction of the object.

\section{Monitoring of arterial pulse wave and oxygen saturation}

The most common example of transillumination is the observation of arterial blood pulsation allowing for measuring the blood pulse and blood oxygenation $[2,10]$. Measurements of arterial blood gases and $\mathrm{pH}$ provide information about the adequacy of blood oxygenation and $\mathrm{CO}_{2}$ elimination. Knowing what percentage of the hemoglobin is saturated with oxygen is important when administering anesthesia as well as for helping diagnose various diseases.

All traditional in vitro measurements of blood oxygen content need to draw blood samples are risky, rich in complications and very time consuming. Early evaluations of blood oxygenation with noninvasive, transcutaneous techniques based on light-tissue interaction were unacceptable in practice; however, the progress which has been made in this area is excellent. Credit for the great present interest in these measurements belongs to anesthesiologist William New who with engineer Jack Lloyd founded in USA Nellcor Incorporated [11] where first commercial device called pulse oximeter was fabricated in 1985. Today, various models are manufactured and used in a lot of countries in the world. However, up to now, the method employed is a subject of continuous development and improvement.

\subsection{Photoplethysmography and pulse oximetry}

The modulations of biooptical signals, induced in tissues by the arterial blood pulsations, are the basis of very important optical blood-less diagnostic methods: photoplethysmography (PPG) and pulse oximetry $[2,8]$ which make possible to monitor noninvasively the living tissues vitality. The analysis of PPG with the simultaneous monitoring of ECG makes possible to decrease influence of artifacts, and produce accurate results in patients with often poor peripheral 
circulation. Pulse oximetry which is currently known as one of the most significant advance made in patient monitoring, bases on noninvasive use of two phenomena: natural arterial pulsations and differences in optical properties of blood and other tissues. This technique smartly joins rules of both in vivo spectrophotometry and photoplethysmography to monitor the arterial blood oxygen saturation $\mathrm{SaO}_{2}=\mathrm{HbO}_{2} /\left(\mathrm{Hb}+\mathrm{HbO}_{2}\right)$ (fig. 2). What is unique in pulse oximetry that it is possible to sense the global oxygen saturation of human body arterial blood by noninvasive transillumination of only a peripheral tissue set which allows us to see a "representative" arterial blood in other tissue components. The principle is simple as based on spectrophotometry rules, referring to idealized blood consisting of only two absorbers to be detected.
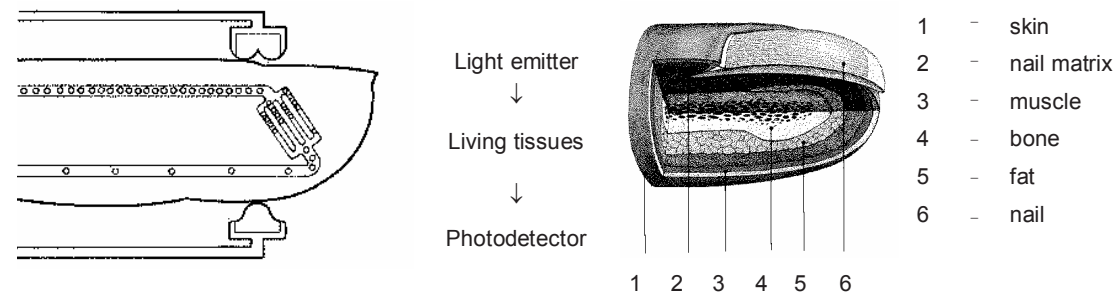

Cross-section of the fingertip to be transilluminated with an optoelectronic sensor

PPG signal from the peripheral body site:

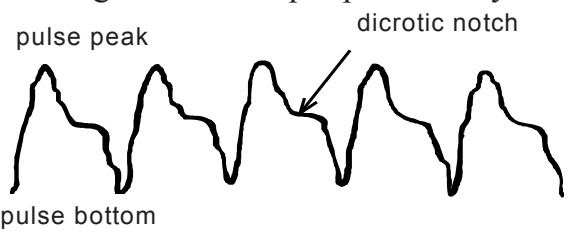

Biooptical phenomena concerning with hemoglobin:

Molar absorption coefficient

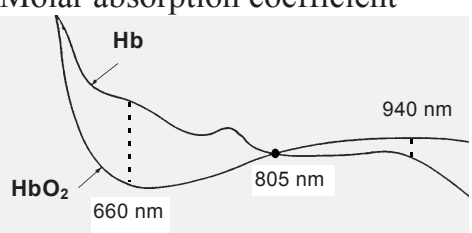

Wavelength $\lambda$
The percentage oxygen saturation of arterial blood:

$$
\mathrm{SaO}_{2}=\mathrm{HbO}_{2} /\left(\mathrm{Hb}+\mathrm{HbO}_{2}\right)
$$

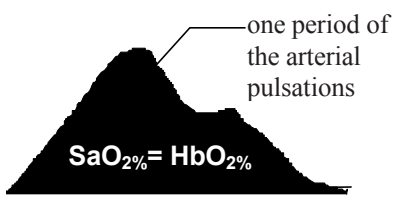

Numerical indication of pulse oximeter: $\mathrm{SpO}_{2 \%}=\mathrm{SaO}_{2 \%} \pm \Delta \%$

Figure 2: Illustration of PPG and pulse oximetry measurement signals and arrangement of a transmission noninvasive optoelectronic sensor placed on the fingertip - a pulse oximeter indication $\mathrm{SpO}_{2} \%$ estimates the true value $\mathrm{SaO}_{2} \%$ with the uncertainty $\Delta_{\%}$. 
In practice, the measurements are accomplished with optoelectronic sensors placed on "living cuvettes" such as, e.g., finger-tips, ear-lobes, nasal bridges. The finger-tips are the especially useful sites to place the sensors which are applied directly and very often in prolonged duration. During laboratory studies, the authors of this paper use the modern devices shown in fig. 3 .
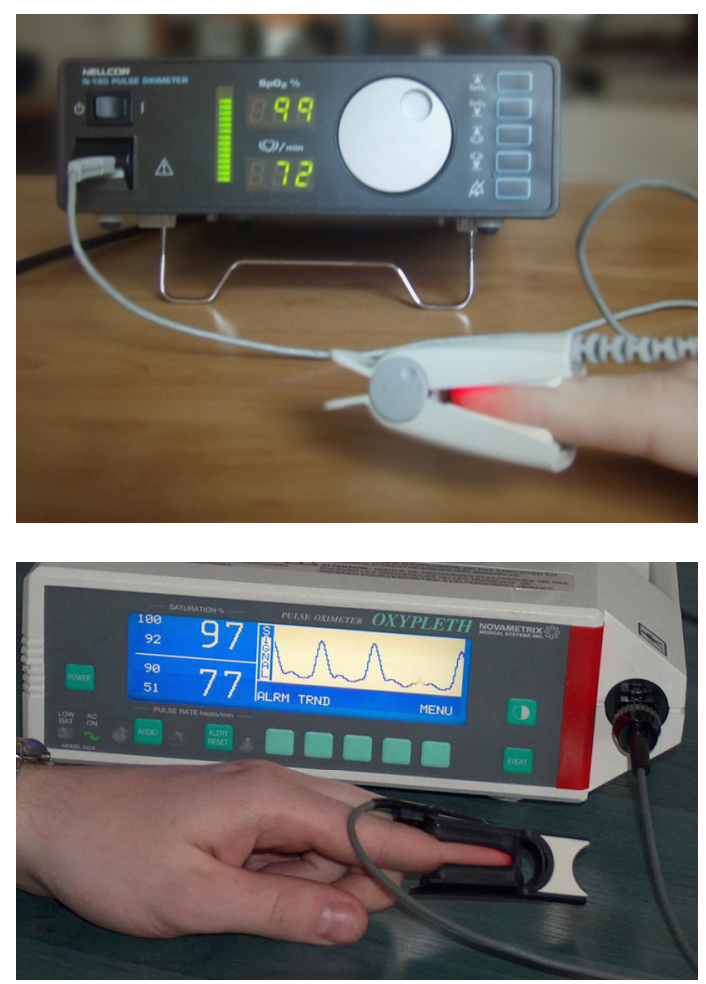

Figure 3: Examples of the measurement results obtained by the authors during their studies with two models of pulse oximeters: Nellcor180 (top), and Oxypleth Novametrix (bottom). The latter is equipped with a PPG waveform monitoring system.

The majority of the manufactured commercial pulse oximeters, which are portable models or of pocket size units, display the values of oxygen saturation and pulse rate, while the other can display pulse strength, present the trend from a past period of time, and to display specific waveforms. The models which are only slightly larger than most the reusable finger sensors, have been designed especially for evacuation situations. The alarms mean the critical functions in a pulse oximeter, alerting of a potentially dangerous situations that usually include: high oxygen saturation, low oxygen saturation, high pulse rate, and low pulse rate.

The thickness of the object varies with each pulse, changing the light path length which effects are eliminated in estimating the oxygen saturation. The 
difference in shape and stability of the peripheral photoplethysmographic waveform can be used as an indication of possible motion artifacts or low perfusion conditions (fig. 4). Similarly, if the patient's heart rate displayed by the pulse oximeter differs considerably from the actual heart rate, the displayed saturation value should be questioned.

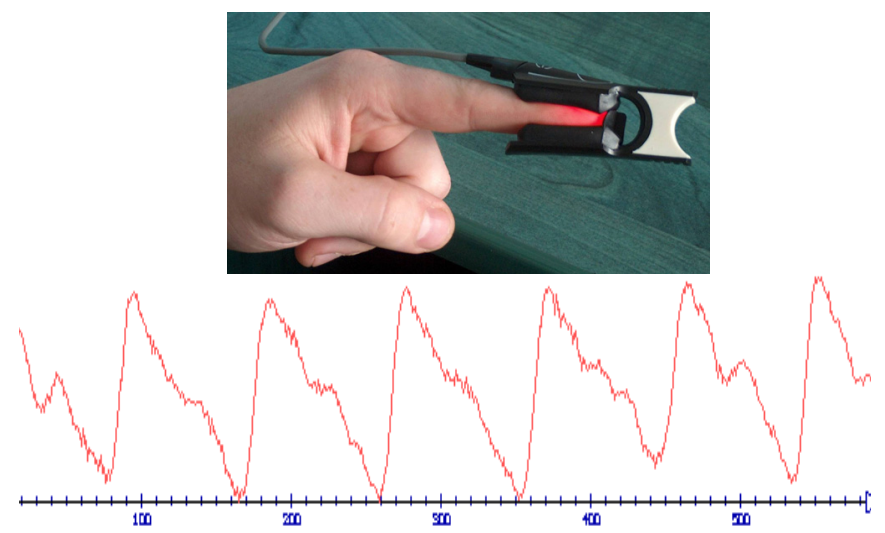

Figure 4: Example of a raw pulse wave acquired from the finger sensor.

\subsection{Novel expanded use of the transmission variant of pulse oximetry concept}

A modified approach to the transmission variant of pulse oximetry that was used by the authors in their own studies [12] depends on controlling tendency to changes in the selective components of light transmitted by an examined body site. The components of two raw pulsatile optical signals from the object (fig. 5),

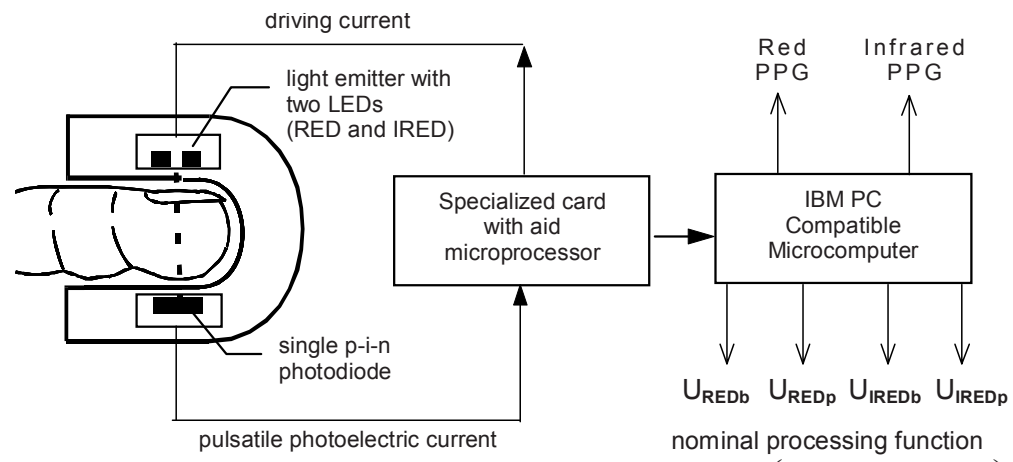

$$
\mathrm{SpO}_{2}=F\left(\frac{\mathrm{U}_{\text {REDp }} / \mathrm{U}_{\text {REDb }}}{\mathrm{U}_{\text {IREDp }} / \mathrm{U}_{\text {IREDb }}}\right)
$$

Figure 5: Scheme of the testing device based on a novel use of the pulse oximetry concept. 
to be obtained at each of both wavelengths (i.e. $U_{\text {RED }}$ and $U_{\text {IRED }}$ ), can be processed to four values of voltage which correspond to a reference bottom $\left(\mathrm{U}_{\mathrm{REDb}}, \mathrm{U}_{\text {IREDb }}\right)$, and a peak difference of the arterial pulse wave $\left(\mathrm{U}_{\text {REDp }}, \mathrm{U}_{\text {IREDp }}\right)$ in each heart beat. Output signals from the sensor are held in the desirable range of values by assignment of current pulses which control the photoemitter. The real relationship between a ratio: $\left(\mathrm{U}_{\mathrm{REDp}} / \mathrm{U}_{\mathrm{REDb}}\right) /\left(\mathrm{U}_{\text {IREDp }} / \mathrm{U}_{\text {IREDb }}\right)$ and $\mathrm{SpO}_{2}$ which values estimate $\mathrm{SaO}_{2}$, has been established experimentally.

A novelty is continuous tracing the components of optical signals acquired from the transilluminated object. Indicating pulse waveforms and changes in the raw PPG signals at both measuring wavelengths allow the end-user to assess in real time the quality and reliability of the measurements. Firstly, the shape and stability of the photoplethysmographic curve can be used as an indication of possible influences. Secondly, by controlling a tendency to changes in raw values of quantities involved in the known nominal processing function, falsely true readings have potential to be detected and corrected.

On the one hand, studying the relationships between either pathological changes in a circulatory rhythm or measurement disturbances and corresponding to them changes in the frequency spectrum of the photoplethysmogram detected from a peripheral body site may be useful in practice. On the other hand, computer creation and analysis of virtual pulse waveforms can be useful to simulate real signals occurring in pathological situations when measurements are impossible because of either ethical or technical limitations. The obtained results may be useful in computer-aided generation of reference data for evaluation of light-tissue spatial transillumination. Computer models in particular have been increasingly successful in simulating bio-optical phenomena.

\section{Optical imaging of hand fingers}

In 1895 Wilhelm Conrad Roentgen made the first radiogram of a palm, starting the development of noninvasive image diagnostics methods. Currently, different imaging methods are able to detect different properties of investigated tissues through a variety of phenomena to be utilized. The role of optical techniques in current tendency to developing combined medical imaging is especially significant in such a field as, e.g., the modern videoendoscopy [5].

Light scattering and absorption can complicate the transillumination image. The optical image resolution can be lower than that of X-ray images, however, it enables to provide information on the functional conditions unavailable in the RTG technique.

The experimental transillumination of hand and foot fingers is possible in a quite simple as well as quite efficient transmitting-receiving system presented in fig. 6 [13]. The mechanical structure of this system was constructed in the form of letter C fixed to the robot's arm. A LED diode and photodiode were fit at the structure ends in optical channels of $3 \mathrm{~mm}$ diameter and about $20 \mathrm{~mm}$ length.

The robot arm assembly is flexible and contains the motorization, brakes, motion transmission mechanisms, cable bundle, pneumatic and electrical circuits for the user. A scanning system has been joined with the robot arm. The object's 
scanning is made in a rectangular $x-y$ coordinates system. The hand examined is laid on a transparent plate stabilizing its position. A tissue volume is represented by its thickness in the direction of the incident light action. Various high power LEDs were used in the tests. The optical part of the transmitting-receiving system is LED diode placed opposite the PIN photodiode PD. Several wavelengths were used for the tests. The results presented herein have been obtained for an ELJ-880-228B emitting light at the wavelength $\lambda=880 \mathrm{~nm}$. This LED was driven with current impulses of parameters as follows: $I_{m}=7 \mathrm{~A}, T_{\mathrm{i}}=1 \mu \mathrm{s}$, $T_{\text {rep }}=1 \mathrm{~ms}$. The PIN BPW24R photodiode was used as the sensitive photodetector.

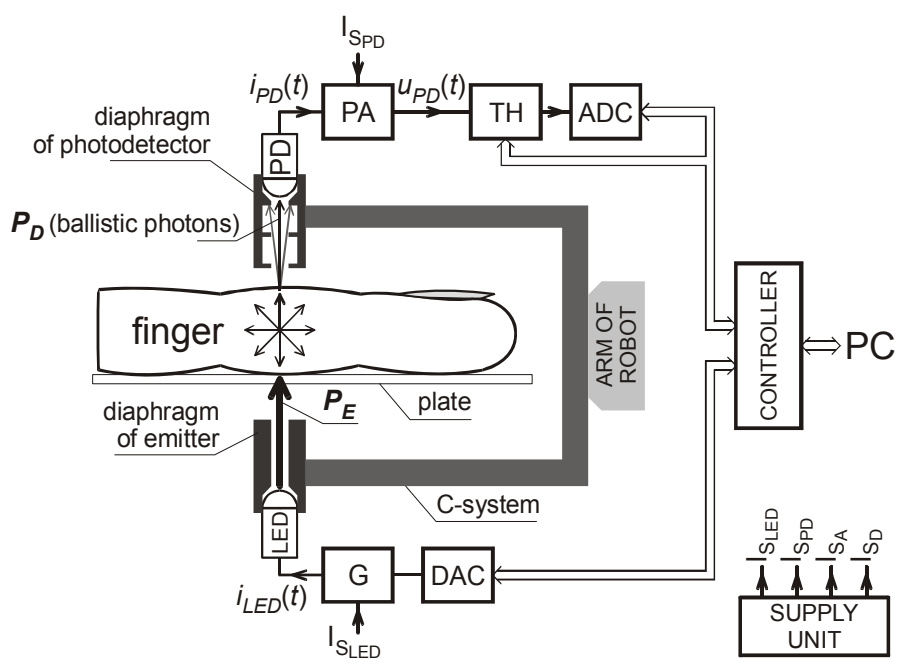

Figure 6: System performed for the impulse transillumination of a finger: PD - photodiode (as photodetector), PA - amplifier and converter of photocurrent $i_{P D}(t), \mathrm{TH}$ - trace and hold converter, ADC - analogto-digital converter, LED - photoemitter, G - source of current pulses $i_{L E D}(t)$, DAC - digital-to-analog converter, $P_{E}-$ radiation illuminating the object, $P_{D}-$ radiation detected by photodiode PD.

The current signal $i_{P D}(t)$ was converted to the corresponding voltage signals and amplified. The voltage $u_{P D}(t)$ appearing during generation of an optical impulse is then subject to analog-to-digital conversion. Reciprocal of the obtained values, marked as $U_{M}$, were collected for particular positions of the scanning system. The relationship occurring between values of $U_{M}$ and levels of $P_{D}$ signal power shows differences in light attenuation connected with absorption and scattering of the transilluminated object. High values of $U_{M}$ prove low levels of $P_{D}$ signal power - in other words such relation shows strong attenuation for a given position. Figure 7 presents the standardized values of the converted output signal from the photodiode for several $x$ values. 


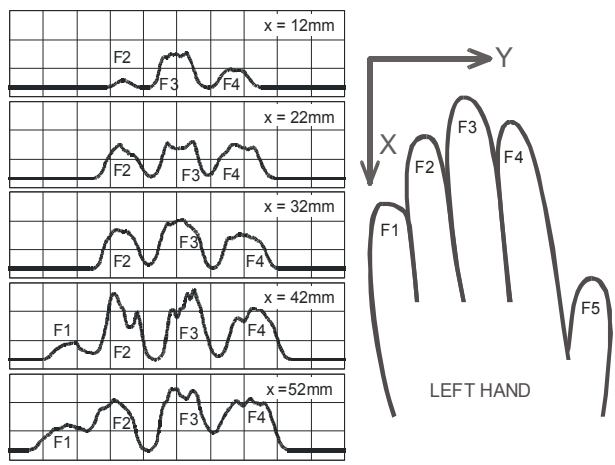

Figure 7: $\quad$ Specification of the output signal dependency on the $y$ location for selected cross-sections with $x$ coordinate.

The results obtained as preliminary transillumination images are shown in fig. 8 . The grayness intensity is represented by the output signal values of the fingers examined (F1, F2, F3, F4). The specific isolines illustrate transmission properties of fingers at the used optical radiation. Despite the measuring system simplicity the imaging obtained was as anticipated. For example, differences between the amplitude variability for fingers without and with joint degenerations were observed. To transilluminate some optically thicker body parts it is necessary to increase the system's sensitivity, better concentration of the optical bundle and possibility to select the detectable photons. The scanner is being built to achieve this goal.
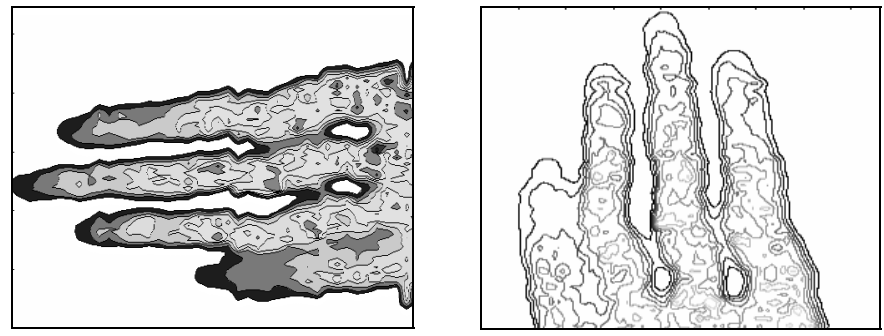

Figure 8: Examples of transillumination images obtained for hand fingers.

\section{Conclusion}

All traditional in vitro biomeasurements that need to draw blood or other tissue samples are risky, rich in complications and very time consuming. From this point of view, the role of noninvasive optical techniques in current medical diagnostics is significant, however, numerous issues related to the result interpretation still need to be solved.

The authors of the paper concentrate their current research on mastering efficient transillumination of thick layers of tissues and building efficient and 
stable algorithms representing the anatomic and functional properties. Transillumination as the method of examination by the passage of light through tissues or a body cavity is a diagnostic technique in the course of intensive development at the moment. From the combined imaging point of view, the optical imaging may provide information on the functional condition unavailable in X-ray and other techniques. Of course progressive development of noninvasive optical imaging and measurements always depends very much on the clinical acceptance of the advanced biomedical technology and engineering.

\section{References}

[1] Enderle, J., Blanchard, S. \& Bronzino, J., Introduction to biomedical engineering, Academic Press: San Diego, 2000.

[2] Mannheimer, P.D., The light-tissue interaction of pulse oximetry. Anesthesia \& Analgesia, 105(6), pp. 10-17, 2007.

[3] King, R., Optoelectronic sensors in medical applications. Texas Advanced Optoelectronic Solutions (TAOS), Inc, September 2003.

[4] Perez, R.J., Optical sensors (Chapter 8). Design of medical electronic devices, Academic Press: San Diego, 2002, pp. 237-73.

[5] Cysewska-Sobusiak, A., Wiczynski, G., Krawiecki, Z. \& Sowier A., Role of optical techniques in combined use of selected methods of medical imaging. Opto-Electronics Review, 16(2), pp. 136-146, 2008.

[6] Duck, F.A., Physical properties of tissue: a comprehensive reference book, Academia Press: San Diego, 1990.

[7] Cysewska-Sobusiak, A., One-dimensional representation of light-tissue interaction for application in noninvasive oximetry. Optical Engineering, 36, pp. 1225-1233, 1997.

[8] Cysewska-Sobusiak, A., Powers and limitations of noninvasive measurements implemented in pulse oximetry. Biocybernetics and Biomedical Engineering, 22, pp. 79-96, 2002.

[9] Pogue, B.W., McBride, T.O., Osterberg, U.L. \& Paulsen, K.D., Comparison of imaging geometries for diffuse optical tomography of tissue. Optics Express, 4(8), pp. 270-286, 1999.

[10] Webster, J.G., Design of pulse oximeters, Institute of Physics Publishing: Bristol and Philadelphia, 1997.

[11] Nellcor technology overview: Nellcor Symphony N-3000 - The next generation on Nellcor Pulse Oximetry. Nellcor: Pleasanton, 1995.

[12] Cysewska-Sobusiak, A., Wiczynski, G. \& Jedwabny, T., Specificity of software co-operating with an optoelectronic sensor in a pulse oximeter system. Proc. SPIE, 2634, pp. 172-178, 1995.

[13] Cysewska-Sobusiak, A. \& Wiczynski, G., Preliminary results of using transillumination for optical imaging of hand fingers. Proc.of the Symposium on Photonics Technologies for the $7^{\text {th }}$ Framework Program, Wroclaw, Poland, pp. 333-336, 2006. 